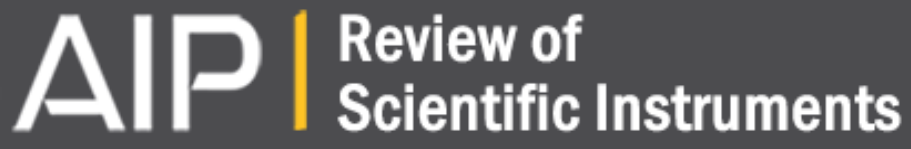

\section{High resolution, low $\mathrm{h} v$ photoelectron spectroscopy with the use of a microwave excited rare gas lamp and ionic crystal filters}

S. Suga, A. Sekiyama, G. Funabashi, J. Yamaguchi, M. Kimura, M. Tsujibayashi, T. Uyama, H. Sugiyama, Y. Tomida, G. Kuwahara, S. Kitayama, K. Fukushima, K. Kimura, T. Yokoi, K. Murakami, H. Fujiwara, Y. Saitoh, L. Plucinski, and C. M. Schneider

Citation: Review of Scientific Instruments 81, 105111 (2010); doi: 10.1063/1.3488367

View online: http://dx.doi.org/10.1063/1.3488367

View Table of Contents: http://scitation.aip.org/content/aip/journal/rsi/81/10?ver=pdfcov

Published by the AIP Publishing

\section{Articles you may be interested in}

An experimental setup for high resolution $10.5 \mathrm{eV}$ laser-based angle-resolved photoelectron spectroscopy using a time-of-flight electron analyzer

Rev. Sci. Instrum. 82, 095113 (2011); 10.1063/1.3637464

Important Atomic, Molecular and Radiative Processes in Low Pressure Discharge Lamps AIP Conf. Proc. 926, 281 (2007); 10.1063/1.2768860

Thermodynamics and microstructure of vacancies in rare gas crystals at high temperature Low Temp. Phys. 33, 578 (2007); 10.1063/1.2755184

Experimental and theoretical study of a differentially pumped absorption gas cell used as a low energy-pass filter in the vacuum ultraviolet photon energy range

J. Vac. Sci. Technol. A 18, 2533 (2000); 10.1116/1.1288196

Vacuum ultraviolet rare gas excimer light source

Rev. Sci. Instrum. 68, 1360 (1997); 10.1063/1.1147942

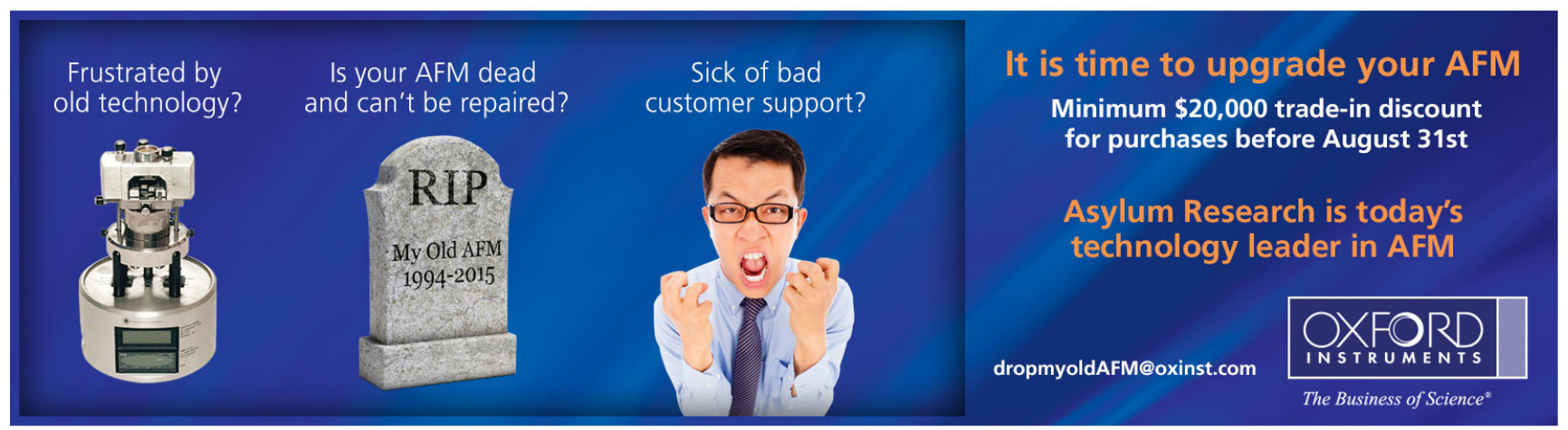




\title{
High resolution, low $h \nu$ photoelectron spectroscopy with the use of a microwave excited rare gas lamp and ionic crystal filters
}

\author{
S. Suga, ${ }^{1, a)}$ A. Sekiyama, ${ }_{1}^{1}$ G. Funabashi, ${ }^{1}$ J. Yamaguchi, ${ }^{1}$ M. Kimura, ${ }^{1}$ M. Tsujibayashi, ${ }^{1}$ \\ T. Uyama, ${ }^{1}$ H. Sugiyama, ${ }^{1}$ Y. Tomida, ${ }^{1}$ G. Kuwahara, ${ }^{1}$ S. Kitayama, ${ }^{1}$ K. Fukushima, ${ }^{1}$ \\ K. Kimura, ${ }^{1}$ T. Yokoi, ${ }_{3}^{1}$ K. Murakami, ${ }^{1}$ H. Fujiwara, ${ }^{1}$ Y. Saitoh, ${ }^{2}$ L. Plucinski, ${ }^{3}$ \\ and C. M. Schneider ${ }^{3}$ \\ ${ }^{1}$ Graduate School of Engineering Science, Osaka University, 1-3 Machikaneyama, Toyonaka, \\ Osaka 560-8531, Japan \\ ${ }^{2}$ Synchrotron Radiation Research Center, Japan Atomic Energy Agency, Hyogo 679-5148, Japan \\ ${ }^{3}$ Institute of Solid State Research-Electronic Properties (IFF-9), Research Center Jülich, \\ Jülich 52425, Germany
}

(Received 28 July 2010; accepted 18 August 2010; published online 22 October 2010)

\begin{abstract}
The need for not only bulk sensitive but also extremely high resolution photoelectron spectroscopy for studying detailed electronic structures of strongly correlated electron systems is growing rapidly. Moreover, easy access to such a capability in one's own laboratory is desirable. Demonstrated here is the performance of a microwave excited rare gas ( $\mathrm{Xe}, \mathrm{Kr}$, and $\mathrm{Ar}$ ) lamp combined with ionic crystal filters (sapphire, $\mathrm{CaF}_{2}$, and $\mathrm{LiF}$ ), which can supply three strong lines near the photon energy of hnyu $\mathrm{h} \nu=8.4,10.0$, and $11.6 \mathrm{eV}$, with the $\mathrm{h} \nu$ resolution of better than $600 \mu \mathrm{eV}$ for photoelectron spectroscopy. Its performance is demonstrated on some materials by means of both angle-integrated and angle-resolved measurements. (C) 2010 American Institute of Physics. [doi:10.1063/1.3488367]
\end{abstract}

\section{INTRODUCTION}

Photoelectron spectroscopy (PES) is a very powerful means to enable the direct probe of electronic structures of materials. This technique has gradually been advanced in the past decades with the development of light sources and the improvement in electron analyzers. In particular, dramatic developments are achieved with respect to the energy resolution and the energy range to be covered. For example, synchrotron radiation (SR) from undulators has realized high brilliance, which facilitates studies of small size materials with good statistics with the use of a well focused SR spot. The linear polarization of the bending SR and planarundulator SR, as well as the circular polarization of the off orbital-plane bending SR and helical-undulator SR, promoted symmetry analyses of the electronic structures as well. ${ }^{1}$

It is nowadays well recognized that the photoelectron kinetic energy $\left(E_{K}\right)$ in the range of $10-200 \mathrm{eV}$ corresponds to the minimum of its inelastic mean free path $(\lambda)$ lying in the range of 3-5 A, realizing rather high surface sensitivity. This characteristic is very convenient for general surface sciences. However, the electronic structures in the bulk can be different from those in the surface region if (1) there is surface reconstruction and/or relaxation or (2) electron correlation effects are not negligible. The latter case is the natural consequence of the reduction of the band width $\mathrm{W}$ near the surface, where the ratio of $\mathrm{U} / \mathrm{W}$ ( $\mathrm{U}$ is the on-site electron Coulomb repulsive energy) becomes noticeably enhanced.

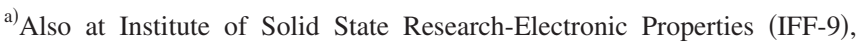
Research Center Jülich, 52425 Jülich, Germany. Electronic mail: ssmsuga@gmail.com.
}

Then, the electronic structures become often less itinerant near the surface in the case of strongly correlated electron systems (SCES), as generally known from theories. ${ }^{1}$ Such less itinerant electronic structures in the surface region are mostly probed by the abovementioned conventional photoelectron spectroscopy. In order to overcome the surface sensitivity of this PES technique for SCES, higher $\mathrm{E}_{\mathrm{K}}$ soft $\mathrm{X}$-ray PES (SXPES) with high energy resolution has first been developed, demonstrating the importance of probing bulk electronic structures in $\mathrm{SCES}^{2-4}$ by this approach. Although SXPES with $\lambda$ in the region of 5-15 $\mathrm{A}$ is found to be very powerful, it has still been gradually recognized as even the SXPES has the surface spectral weight up to $50 \%$ in some cases. Then, higher $\mathrm{E}_{\mathrm{K}}$ hard $\mathrm{X}$-ray PES named HAXPES is understood to be more favorable for probing bulk electronic structures of SCES. ${ }^{5,6}$ However, the photoionization cross sections become orders of magnitude decreased with increasing $\mathrm{h} \nu$ from 1 to $8-10 \mathrm{keV}^{7}$ In spite of the higher possible $\mathrm{h} \nu$ resolution, the resulting total resolution of HAXPES is often spoiled by the single nucleus recoil effects. ${ }^{8-10}$ Therefore, extremely high energy resolution bulk sensitive HAXPES and very reliable band mapping by hard x-ray angle resolved PES (HAX-ARPES) might be difficult in some cases.

Nevertheless, such a light source with high photon flux and high resolution in $\mathrm{h} \nu$ is generally required to perform high quality PES. In the past decade, the low energy PES excited by a quasi-cw laser was eagerly pursued and turned out to be bulk sensitive in some cases. ${ }^{11-13}$ If the pulse width is too short, however, the uncertainty principle will induce the energy broadening. If the laser power density is too high, the space charge effects will also spoil the resolution. Therefore, quasi-cw laser with high enough repetition and with 


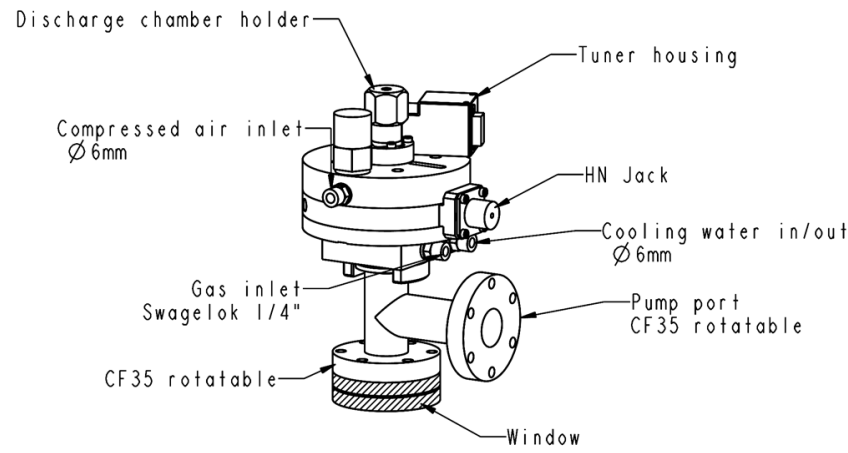

FIG. 1. Drawing of the T-1 lamp head. The bottom window is replaced by a filter-mirror chamber, as later illustrated in Fig. 4.

reasonably long pulse width is required for the purpose of extremely high resolution $\left(\Delta \mathrm{E}_{\mathrm{K}}\right)$ PES. Since the work functions of many solids are near $5 \mathrm{eV}$, the $\mathrm{h} \nu$ slightly above 6-7 eV will be mostly desirable. However, such light sources compatible with these requirements are rather limited at present. Any easily accessible light source satisfying these requirements is highly demanded for general scientific purposes in the recent years. The discharge lamps by $\mathrm{He}, \mathrm{Ne}$, and other rare gases were traditionally used for PES excitations. However, the $\mathrm{h} \nu$ 's of $\mathrm{He}$ and $\mathrm{Ne}$ are too high and realize the surface sensitive $E_{K}$ for the electrons in SCES near the Fermi level $\left(\mathrm{E}_{\mathrm{F}}\right)$. The self-absorption and Dopplereffect induced broadening could not realize the $h \nu$ resolution better than $1 \mathrm{meV}$. Lower pressure and slower thermal motion of rare gas atoms are required for realizing the extremely narrow linewidth of the rare gas light source. This problem is now technically solved by employing the microwave excited rare gases loaded in a small ceramic tube under an external magnetic field. The $\mathrm{h} \nu$ resolution better than $600 \mu \mathrm{eV}$ is achieved for the strongest lines of these gases at three different $\mathrm{h} \nu,{ }^{14}$ which can be easily extracted with high efficiency by using proper ionic crystal filters. Details of the systems and typical examples of the experimental results are reported in this paper.

\section{LIGHT SOURCE AND OPTICAL SYSTEM}

The sketch of the light source (T-1 lamp head made by MB Scientific AB) is given in Fig. 1. Its cross section is given in Fig. 1 of Ref. 15, where a ceramic tube is vertically sandwiched between the discharge-chamber holder and the light exit aperture facing toward the T-type nipple. The microwave is transferred from the microwave generator via a coaxial-cable-type waveguide and input to the lamp head via a $\mathrm{HN}$ jack. A magnetic field is applied perpendicularly to the axis of the ceramic tube. The ceramic tube is air-cooled and the lamp head with the light emission exit aperture is watercooled during the operation. The gas excited by the microwave in this small ceramic tube by means of the electroncyclotron resonance plasma emits lights through a metal aperture of $2 \mathrm{~mm}$ in diameter. $\mathrm{Xe}, \mathrm{Kr}$, or Ar gas is supplied into this ceramic tube with a length of $\sim 50 \mathrm{~mm}$ and an inner diameter of $\sim 4 \mathrm{~mm}$ through the gas inlet. The gas pressure is kept in the middle $10^{-3}$ Torr range during the operation. Although a mass flow meter is very useful for a stable op-

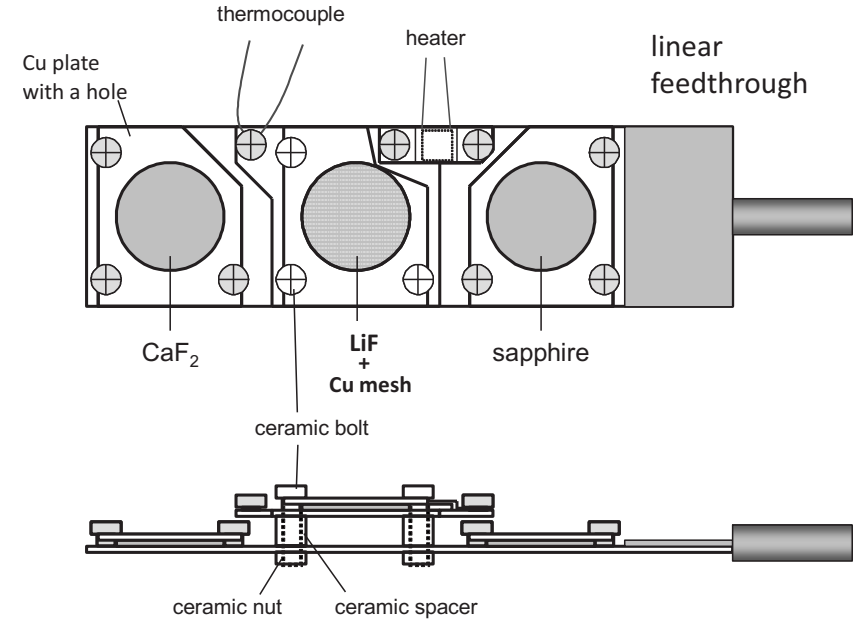

FIG. 2. Mounting of the sapphire, $\mathrm{CaF}_{2}$, and $\mathrm{LiF}$ filters on a $\mathrm{Cu}$ substrate attached to an in-vacuum linear feedthrough. Heating and temperature control are necessary for the LiF filter for the use of an Ar lamp.

eration, a variable leak valve set to a certain level in the upstream position of the gas inlet is enough for an operation over several hours. The gas supplied through the variable leak valve is pumped down by a turbomolecular pump in the downstream position of the light exit aperture. Unnecessary gas should be pumped down as much as possible to reduce the self-absorption and realize narrower energy width of the strongest emission line. The exchange of the gas species can be easily realized within about $10 \mathrm{~min}$ by closing the shut valve for the gas to be no more used, pumping the residual gas, opening the proper shut valve for the next gas, and readjusting the variable leak valve. The bottom window in Fig. 1 is replaced by a filter-mirror chamber as explained later.

The emitted light is composed of many lines at different $\mathrm{h} \nu$. Since a monochromatic light is necessary for high accuracy photoelectron measurements, one should use either a grating or a filter to extract the component to be used for the photoelectron excitation. With the use of a grating, one can extract a certain line among several emission lines by mechanically rotating the grating in accordance with the grating equation. Since the light is separated in this case into the zero order light (specular reflection), the first order light, which is used for the measurement and higher order lights, the best designed grating is required to have the highest possible photon flux at the designed $\mathrm{h} \nu$. If one wants to simply use the strongest line from $\mathrm{Xe}, \mathrm{Kr}$, and $\mathrm{Ar}$ near 8.4, 10.0, and $11.6 \mathrm{eV}$, it is practical and inexpensive to use ionic crystal filters (sapphire, $\mathrm{CaF}_{2}$, and $\mathrm{LiF}$, respectively) to cut higher $\mathrm{h} \nu$ components by the exciton absorption or the Urbach tail, ${ }^{16}$ where it is well known that one can tune the cutoff $h \nu$ by changing the temperature of the ionic crystal. We employ high purity sapphire, $\mathrm{CaF}_{2}$, and heated $\mathrm{LiF}$ for extracting the strongest line from these gases ${ }^{14}$ in both Osaka University and Jülich Research Center.

Figure 2 shows the mounting of filters in the system in Osaka University. Three filters are mounted on a $\mathrm{Cu}$ substrate attached to a linear feedthrough. Only the LiF filter is mounted on another $\mathrm{Cu}$ plate isolated from the $\mathrm{Cu}$ substrate by ceramic spacers. A semiconductor heater is used to heat up the $\mathrm{LiF}$ filter and the temperature of the $\mathrm{LiF}$ filter is mea- 


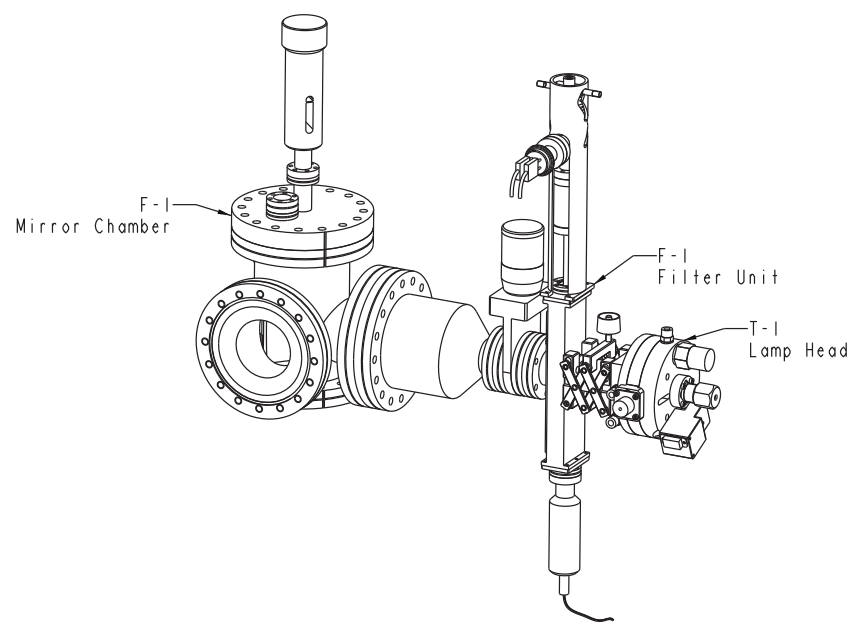

FIG. 3. T-1 lamp head, F-1 filter chamber, and toroidal mirror chamber.

sured by a thermocouple. A heating lamp can also be used to heat up the LiF filter and its temperature is kept constant by a feedback loop. Such a system employed in the system in Jülich is shown in Fig. 3, where the combination of the T-1 lamp and the filter unit (F-1) with the lamp-heating system at the bottom is seen. The light emitted toward left from the lamp passes through the filter and diverges. Then the light is focused by a toroidal mirror in the left chamber.

The whole optical system is schematically shown in Fig. 4. The focusing of the light from the rare gas is quite important in general to enable a measurement of small samples, which may be newly synthesized or have the essential difficulty of increasing their sizes. An incidence angle of $45^{\circ}$ (or $90^{\circ}$ deviation angle) onto the focusing mirror surface is employed in our system to be flexibly adaptable to the photoelectron system with a fixed analyzer, cryostats, and many items for PES measurements. The image distance of the mirror is set to $400 \mathrm{~mm}$. The distance from the mirror center to the light exit aperture (or object distance) is also set to $400 \mathrm{~mm}$ and the one to one focusing is realized. The curvatures (radii) of the toroidal surface are designed as 282.8 and $565.7 \mathrm{~mm}$ for the sagittal and tangential directions, respectively. The light emerging from the aperture of the lamp with a diameter of $2 \mathrm{~mm}$ for a $\pm 5^{\circ}$ cone is focused

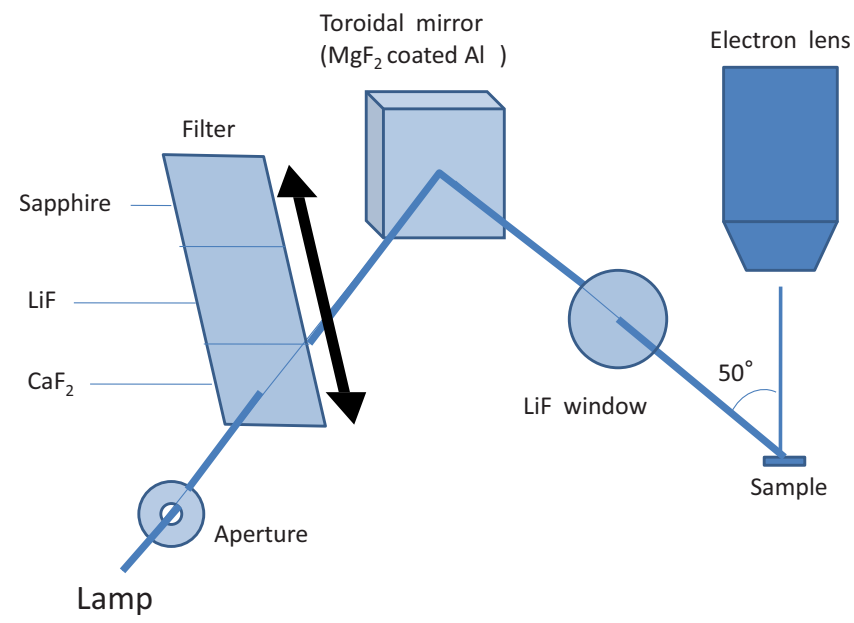

FIG. 4. (Color online) Schematic view of the optical system.

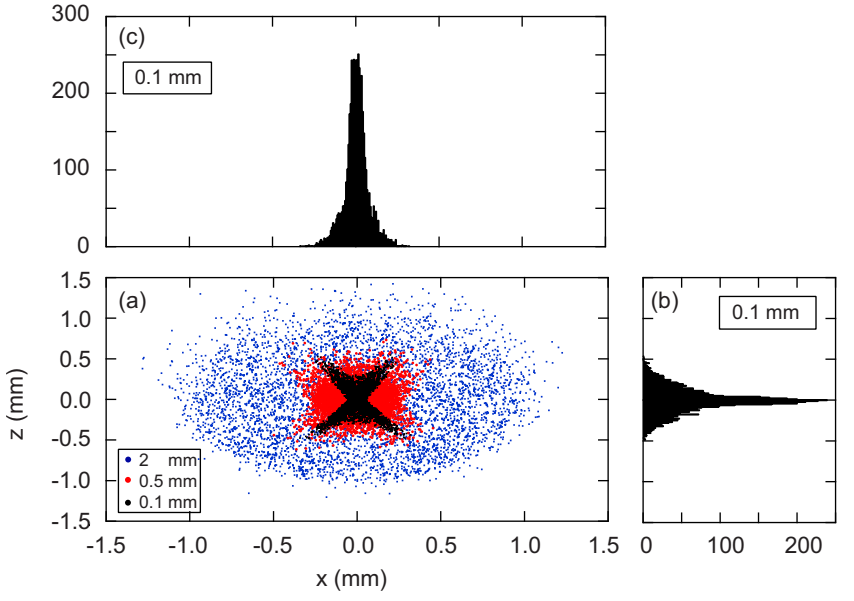

FIG. 5. (Color online) Results of the ray-tracing at the sample position of $400 \mathrm{~mm}$ from the mirror center, which is $400 \mathrm{~mm}$ from the light emitting circular aperture with diameters of 2, 0.5 , and $0.1 \mathrm{~mm}$ (a). The profile of the integrated intensity with respect to the $\mathrm{x}$ and $\mathrm{z}$ directions in the case of $0.1 \mathrm{~mm}$ diameter aperture is shown in (b) and (c). The mirror parameters are given in the text.

onto the sample with the size of $2 \mathrm{~mm}$ as predicted by the results of ray tracing, as shown in Figs. 5(a)-5(c). Here, $x$ and $\mathrm{z}$ axes are the directions perpendicular and parallel to the optical plane, which is defined by the light incidence and reflection directions. Down to the aperture size of $0.5 \mathrm{~mm}$, the aberration by the toroidal mirror is not serious. When the aperture size is further reduced to $0.1 \mathrm{~mm}$, the aberration seems to be no longer negligible. However, the full width at half maximum of the $\mathrm{x}$ and $\mathrm{z}$ integrated intensity can still stay below $0.11 \mathrm{~mm}$ for the $\mathrm{z}$ and $\mathrm{x}$ directions. Since the intensity of the light is proportional to the effective source area given by the light exit aperture, it becomes $\sim 1 / 400$ for the $0.1 \mathrm{~mm}$ aperture. Still, one can see the weak visible part of the light on the sample by the eye and properly position the sample for measurements.

As for the vacuum system, it is noted that a LiF UHV compatible window is employed between the filter-mirror chamber and the analyzer-sample chamber (main chamber) in order to protect the gas condensation onto the sample surface, which is often cooled down to liquid He temperature to reduce the thermal broadening due to the Fermi-Dirac distribution function or to induce the phase transition. By using $\mathrm{LiF}$ window kept at room temperature, one can transmit even the strongest Ar emission line, which is monochromatized by a heated LiF filter, with high efficiency. By virtue of this vacuum isolation, one can measure the clean surface of various transition metal compounds at liquid He temperature without any noticeable degradation over a week, in contrast to the case of He and/or Ne lamp. In order to keep the $\mathrm{LiF}$ window against any mechanical or pressure-difference induced damage, it is supported on both sides by holed grids with the light transmittance of more than $50 \%$. The double sided flange with the $\mathrm{LiF}$ window has a $\mathrm{CF}$ flange on one side facing the main chamber and an O-ring flange on the other side facing the filter-mirror chamber.

The analyzer chamber must be made of micrometal must be replaced by mumetal or in-vacuum shielded by micrometal to minimize the effects of the external magnetic field 
since any residual magnetic field may noticeably distort the orbit of very low $\mathrm{E}_{\mathrm{K}}$ photoelectrons. The sample is first introduced into the air lock chamber by breaking the vacuum and then the chamber is pumped down by a turbomolecular pump. This sample can be transferred onto a cold stage of a closed cycle He cryostat when the top of the cryostat is extracted from the main chamber to the sample preparation chamber and the gate valve to the air lock chamber is opened. The sample mounted on a sample holder plate can be tightly screwed to the cold stage of the He cryostat. Surface ion-sputtering, annealing, and evaporation of $\mathrm{Au}$ and other materials are possible in the sample preparation chamber. In the case of cleavage or fracturing, the sample can be treated either in the sample preparation chamber or in the main chamber at any temperature down to the liquid $\mathrm{He}$ temperature. Another closed cycle He cryostat is fixed to the analyzer chamber. The top of this cryostat has a cylinder made of $\mathrm{Cu}$ and with holes for the light incidence and electron passing. By limiting the opening angle of the holes of this cylinder, sample temperatures down to $3 \mathrm{~K}$ are easily realized and the measurement is possible at any temperature between $3 \mathrm{~K}$ and room temperature with temperature regulation better than $\pm 2 \mathrm{~K}$. The system in Osaka University is constructed as above. Another system in Jülich Research Center is equipped with a liquid $\mathrm{N}_{2}$ cryostat at present, which will be replaced by a liquid He cryostat in the near future.

Combined with this lamp system, we used a SCIENTA SES2002 hemispherical analyzer in Osaka University driven by the MB Scientific A-1 power supply. The minimum slit width installed at present is $0.2 \mathrm{~mm}$. Residual magnetic field is minimized and any insulating material is hidden behind the metallic shield near the sample to not disturb the orbit of emitted photoelectrons for the measurement should be removed since it is repeated of angle resolved measurement. The system in Jülich Research Center is equipped with SES200 hemispherical analyzer. These analyzers are going to be replaced by the MB Scientific A-1 analyzers soon to have wider angular aperture and higher resolving power.

\section{PERFORMANCE}

The transmittance of the ionic crystal filters is summarized in Figs. 2 and 3 of Ref. 15, where one can easily recognize that the temperature control enables the flexible change of the cutoff $h \nu$ of the filter and the extraction of the strongest low $\mathrm{h} \nu$ lines from $\mathrm{Xe}, \mathrm{Kr}$, and Ar lamps. Here, one must remember that the transmittance of the filter is in proportion to $\exp (-t)$, where $t$ is the thickness of the filter. This is the reason why thin filters are required for this purpose. Since the absorption by the exciton Urbach tail is so strong, even thin crystal or thin evaporated film is enough to cut higher $\mathrm{h} \nu$ components.

Although the reflectance of the fresh $\mathrm{Al}$ mirror surface can be quite high, surface oxidation and the resulting reduction of the reflectivity should be protected by coating with $\mathrm{MgF}_{2}$. Since the energy of the band gap and exciton absorption edge of $\mathrm{MgF}_{2}$ is lower than the $11.6 \mathrm{eV}$ emission line of $\mathrm{Ar}$, a thin coating of $\mathrm{MgF}_{2}$ is a necessity for the use of the $\mathrm{Ar}$ emission. We coated $25 \mathrm{~nm}$ of $\mathrm{MgF}_{2}$ in situ onto the fresh $\mathrm{Al}$ surface.

Since the gas pressure is rather low and the excitation is by microwave, the self-absorption and Doppler broadening can be much reduced compared with the conventional rare gas discharge lamp. The resolution of the emission line can be directly checked by using a high resolution grating monochromator. We could evaluate the maximum possible width of the $\mathrm{Xe}$ emission line as $600 \mu \mathrm{eV}$, in which the major part is thought to be resulting from the resolution of the monochromator itself. ${ }^{14}$

The inner part of the ceramic tube becomes gradually black colored and the efficiency of the light emission becomes decreased after its use over thousands of hours. Then, the replacement of the ceramic tube is required, for which less than 10-20 min are required to reignite the lamp. During the use of the ceramic tube, one may experience a slight crack of the ceramic tube. The air leak into the ceramic tube induces additional lines, which can be clearly recognized with the use of Ar $11.6 \mathrm{eV}$ line, since it provides additional PES structures in the lower $E_{K}$ region with the very similar dispersion as the strongly observed electronic states.

The angle resolved photoemission spectra (ARPES) of $\mathrm{Cu}(111)$ clean surface measured at $90 \mathrm{~K}$ by Xe lamp with $\mathrm{LiF}, \mathrm{CaF}_{2}$, and sapphire filters are reproduced in Fig. 6. In comparison with the HAXPES, we employ the names of extremely low energy PES (ELEPES) and its angle resolved mode (ELE-ARPES) hereafter. In the $\mathrm{E}_{\mathrm{K}}$ range of 3.5-3.7 eV is seen a strong band with dispersion in all three cases (three spectra in the lower panel). This band is crossing the $\mathrm{E}_{\mathrm{F}}$. In the case of LiF filter, however, similarly dispersing features are seen up to $7 \mathrm{eV}$, suggesting the presence of emission lines from the Xe lamp above $\mathrm{h} \nu=8.4 \mathrm{eV}$. The dispersing features up to $\mathrm{E}_{\mathrm{K}}=5.3 \mathrm{eV}$ are still seen for the $\mathrm{CaF}_{2}$ filter. However, such dispersive features above $\mathrm{E}_{\mathrm{K}}=4 \mathrm{eV}$ are fully suppressed for the sapphire filter. One also recognizes features without dispersion in the $\mathrm{E}_{\mathrm{K}}$ region of $2-3.2 \mathrm{eV}$ for $\mathrm{LiF}$ and $\mathrm{CaF}_{2}$ filters (three spectra in the lower panel and the top spectra in the upper panel). The absence of dispersion for these features tells us that they are not from the abovementioned structure near the $\mathrm{E}_{\mathrm{F}}$ but from the deeper states with negligible dispersion excited by higher $\mathrm{h} \nu$ emission lines of the Xe lamp. Therefore, such features are absent for the excitation through the sapphire filter as seen in the top panel. Thus, the $\mathrm{E}_{\mathrm{K}}$ region of $1.5 \mathrm{eV}$ down from the $\mathrm{E}_{\mathrm{F}}$ seems to be useful in this case.

The corresponding ELE-ARPES by $\mathrm{Kr}$ lamp is reproduced in Fig. 7 for the $\mathrm{LiF}$ and $\mathrm{CaF}_{2}$ filters. In both cases, a dispersing feature is observed in the range of $\mathrm{E}_{\mathrm{K}}$ $=5.1-5.3 \mathrm{eV}$ near the $\mathrm{E}_{\mathrm{F}}$ for the $\mathrm{h} \nu$ near $10.0 \mathrm{eV}$. An additional feature with similar dispersion is observed near $\mathrm{E}_{\mathrm{K}}$ $=5.8 \mathrm{eV}$ for LiF filter, suggesting that a higher $\mathrm{h} \nu$ photon of about $10.6 \mathrm{eV}$ is also emitted from the $\mathrm{Kr}$ lamp. Two structures, one dispersive around $\mathrm{E}_{\mathrm{K}}=3.3-3.5 \mathrm{eV}$ and the other much less dispersive around $\mathrm{E}_{\mathrm{K}}=3.0 \mathrm{eV}$, are observed for both the $\mathrm{LiF}$ and $\mathrm{CaF}_{2}$ filters. The very weak and dispersive feature observed for the $\mathrm{LiF}$ filter around $\mathrm{E}_{\mathrm{K}}=4 \mathrm{eV}$ is most likely ascribed to the dispersive feature at $\mathrm{E}_{\mathrm{K}}=3.3-3.5 \mathrm{eV}$ excited by the abovementioned weak photon line near 

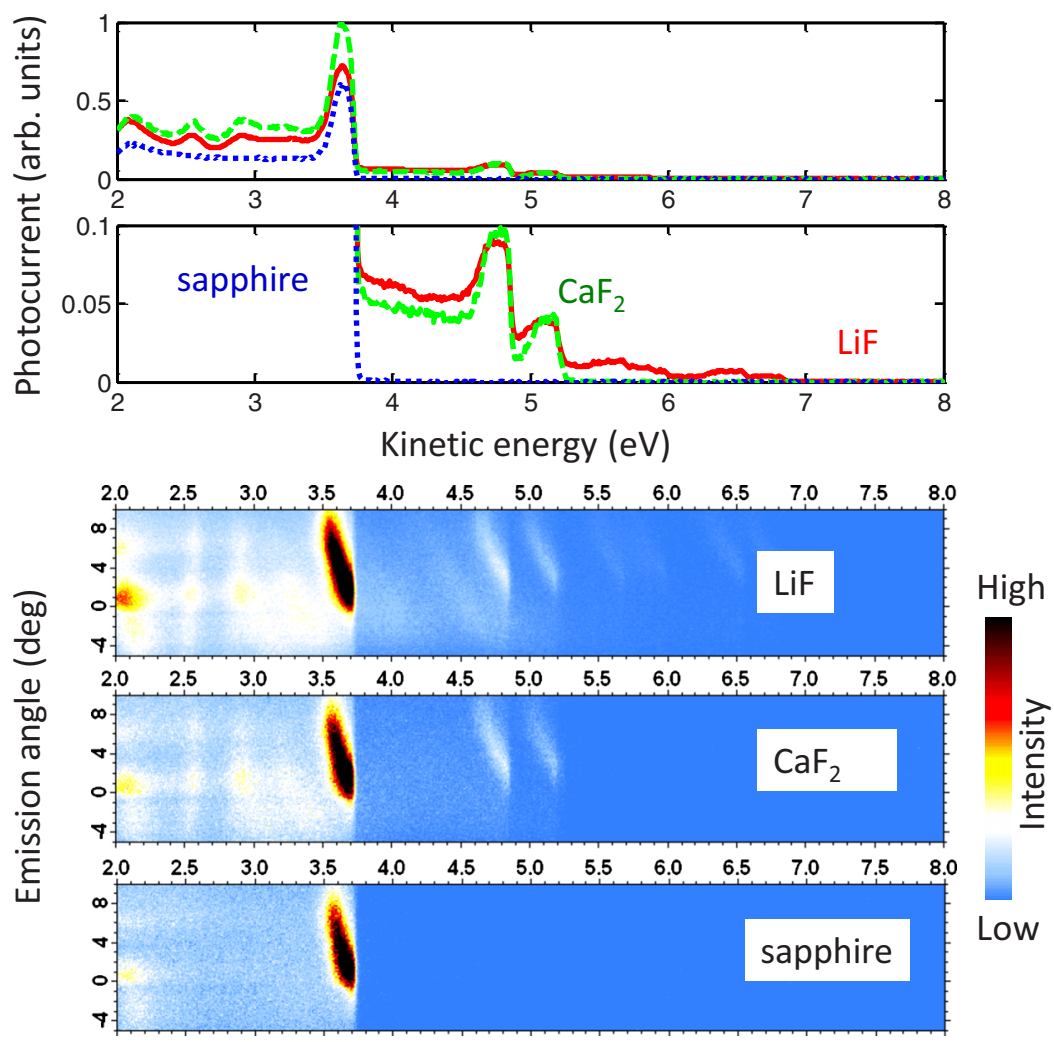

FIG. 6. (Color online) Photoemission spectra of $\mathrm{Cu}(111)$ clean surface at $90 \mathrm{~K}$ excited by the Xe lamp through a $\mathrm{LiF}, \mathrm{CaF}_{2}$, or sapphire filter. The bottom three spectra are ELE-ARPES and the upper two spectra are the ELEPES results in the angle region shown at the bottom three spectra. The scale is ten times magnified in the second top spectra.

$\mathrm{h} \nu=10.6 \mathrm{eV}$. Such a feature is fully suppressed for the $\mathrm{CaF}_{2}$ filter. The three features observed for the $\mathrm{CaF}_{2}$ filter have different behaviors with respect to the dispersions and their wave numbers, suggesting that three electronic states are probed in this measurement. Thus, the $\mathrm{E}_{\mathrm{K}}$ region of $2.4 \mathrm{eV}$ below $\mathrm{E}_{\mathrm{F}}$ can be safely studied by the $\mathrm{Kr}$ lamp $+\mathrm{CaF}_{2}$ filter, which is set at room temperature. When the sapphire filter is used, $\mathrm{h} \nu=10.0 \mathrm{eV}$ photon is suppressed. Even in this case, very weak PES features can be traced in the $\mathrm{E}_{\mathrm{K}}$ region of 2-3.7 eV, as shown in the top panel of Fig. 7, reflecting low $\mathrm{h} \nu$ photons from the $\mathrm{Kr}$ lamp. If comparably weak features excited by the $10.0 \mathrm{eV}$ photons must be discussed under some exceptional circumstances, the safe region will be down to $1.6 \mathrm{eV}$ below the $\mathrm{E}_{\mathrm{F}}$. As seen here, a weak emission is generally present in the lower $\mathrm{h} \nu$ region even after cutting strong higher energy lines. Then, the energy region covered with reliability is limited within $1.5-1.6 \mathrm{eV}$ from the $E_{F}$ in most cases. If one really wants to measure PES down to the lowest possible $\mathrm{E}_{\mathrm{K}}$, the use of grating is sometimes favorable than the use of cutoff filters by exciton absorption.

In the case of the Ar lamp, temperature tuning of the $\mathrm{LiF}$ filter is mandatory to cut the higher $\mathrm{h} \nu$ component from the
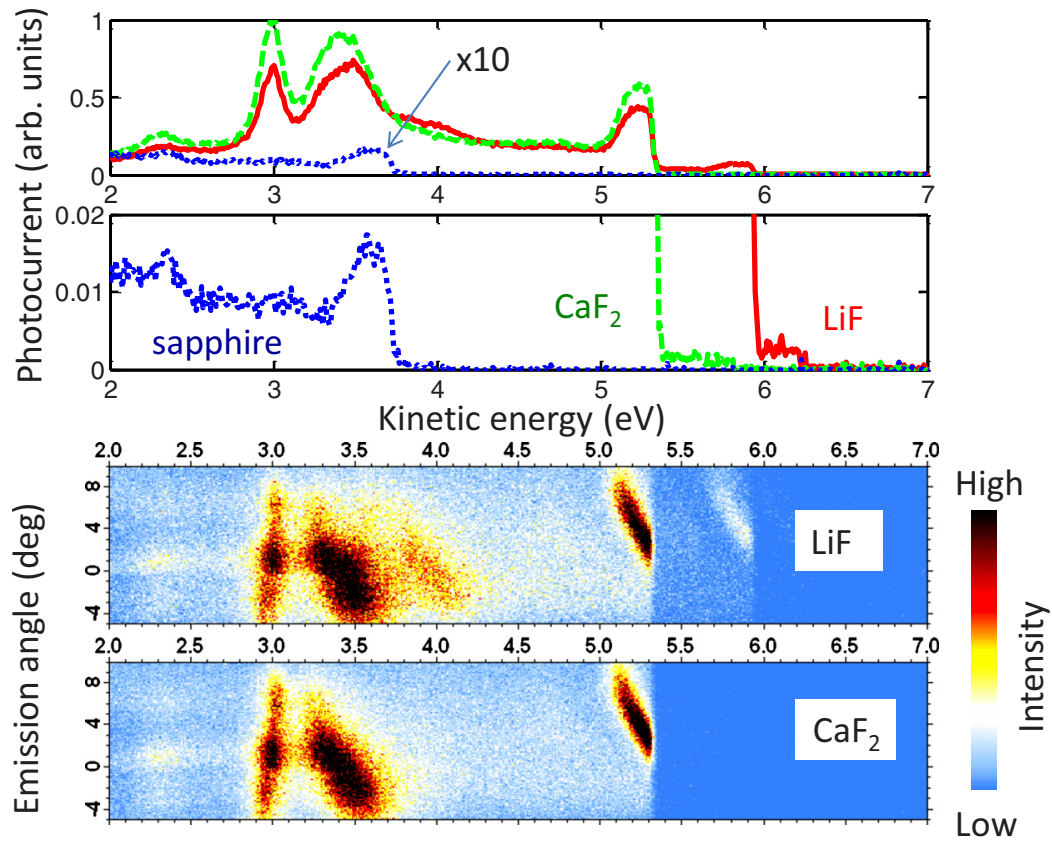

FIG. 7. (Color online) Photoemission spectra of $\mathrm{Cu}(111)$ clean surface at $90 \mathrm{~K}$ excited by the $\mathrm{Kr}$ lamp through the filter. The bottom two spectra are ELE-ARPES for $\mathrm{LiF}$ or $\mathrm{CaF}_{2}$ filter. The upper two spectra are ELEPES results as Fig. 6. The results for the sapphire filter is added just for showing the presence of very low intensity photons from the $\mathrm{Kr}$ lamp even below $\mathrm{h} \nu=8.6 \mathrm{eV}$. In the top panel, the intensity for the sapphire filter is magnified by ten times. In the next panel, all spectra are magnified by 40 times. 


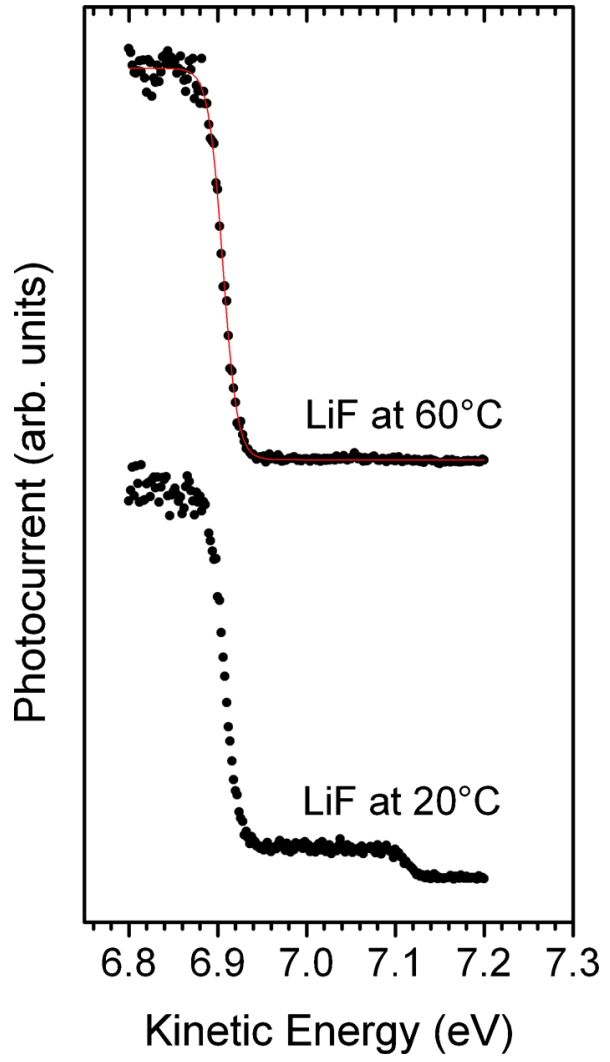

FIG. 8. (Color online) ELEPES by the Ar lamp through a LiF filter at 60 and $20{ }^{\circ} \mathrm{C}$ near the $\mathrm{E}_{\mathrm{F}}$.

lamp. The results on $\mathrm{Cu}$ (111) at $90 \mathrm{~K}$ are shown in Fig. 8, where the weaker Fermi edge at $\mathrm{E}_{\mathrm{K}}=7.1 \mathrm{eV}$ excited by higher $\mathrm{h} \nu$ photons $(\sim 11.8 \mathrm{eV})$ is fully suppressed by the $\mathrm{LiF}$ filter at $60 \mathrm{C}$ (Celsius), leaving the prominent Fermi edge at $\mathrm{E}_{\mathrm{K}}=6.91 \mathrm{eV}$.

\section{SOME APPLICATIONS}

The system in Osaka University was first applied to angle integrated ELEPES measurements of $\mathrm{LiV}_{2} \mathrm{O}_{4}$, which is known as a heavy fermion material. The results at $3 \mathrm{~K}$ are shown in the upper panel of Fig. 9 at $\mathrm{h} \nu=8.4$ and $10.0 \mathrm{eV}$, where the total energy resolution better than $4 \mathrm{meV}$ is achieved. A rather smooth intensity change is observed up to $\mathrm{E}_{\mathrm{F}}$. Although a prominent increase of the PES intensity was observed in the case of $\sim 8 \mathrm{keV}$ HAXPES toward the $\mathrm{E}_{\mathrm{F}},{ }^{17}$ such a behavior is not observed in the ELEPES. In the lower panel as well, one clearly recognizes that all spectra at different temperatures cross the $\mathrm{E}_{\mathrm{F}}$ at the intensity noticeably higher than half of the Fermi edge. This result suggests the presence of higher density of states above the $\mathrm{E}_{\mathrm{F}}$. Since the photoionization cross section $\sigma$ is much stronger for the $\mathrm{O} 2 \mathrm{p}$ component relative to the $\mathrm{V} 3 \mathrm{~d}$ component at these low $\mathrm{h} \nu$, it is understood as the $\mathrm{O} 2 \mathrm{p}$ density of states (DOS) is slightly decreasing toward the $\mathrm{E}_{\mathrm{F}}$, whereas the $\mathrm{V} 3 \mathrm{~d}$ component is increasing toward and beyond the $\mathrm{E}_{\mathrm{F}}$. If the ELEPES is divided by the Fermi-Dirac distribution function (FDDF), DOS can be evaluated beyond the $\mathrm{E}_{\mathrm{F}}$. Then a slight peak is seen in this case around $-10 \mathrm{meV}{ }^{17}$ This peak is ascribed to the DOS of the O 2p states hybridized with the V $3 \mathrm{~d}$ com-

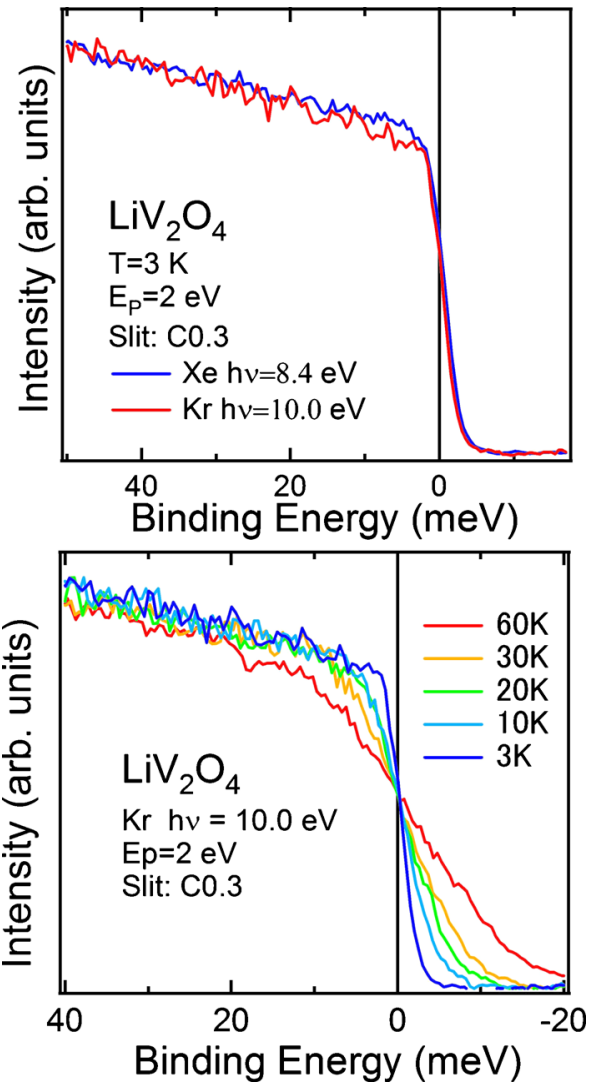

FIG. 9. (Color online) ELEPES of a fractured clean surface of $\mathrm{LiV}_{2} \mathrm{O}_{4}$ at $3 \mathrm{~K}$ excited by $\mathrm{Xe}+$ sapphire or $\mathrm{Kr}+\mathrm{CaF}_{2}$ (upper). The curved $0.3 \mathrm{~mm}$ wide slit and the pass voltage of $2 \mathrm{~V}$ are employed. The temperature dependence is shown in the lower panel.

ponents. Such hybridized states may be responsible for the heavy fermion behavior of this material. ${ }^{12}$ Thus, it is understood that the ELEPES is reflecting the bulk properties in this material.

Next example is for $\mathrm{Yb}_{1-\mathrm{x}} \mathrm{Lu}_{\mathrm{x}} \mathrm{B}_{12}$, which is known as a valence mixed compound with the Kondo temperature of about $80 \mathrm{~K}$. In the spectra shown in Fig. 10, one notices that

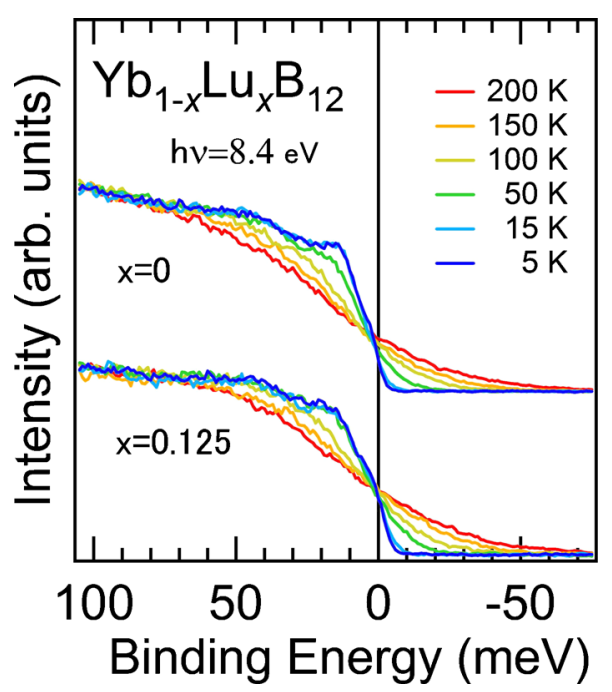

FIG. 10. (Color online) Temperature dependence of the ELEPES of a fractured clean surfaces of $\mathrm{YbB}_{12}$ and $\mathrm{Yb}_{0.875} \mathrm{Lu}_{0.125} \mathrm{~B}_{12}$ excited by $\mathrm{Xe}+$ sapphire. 


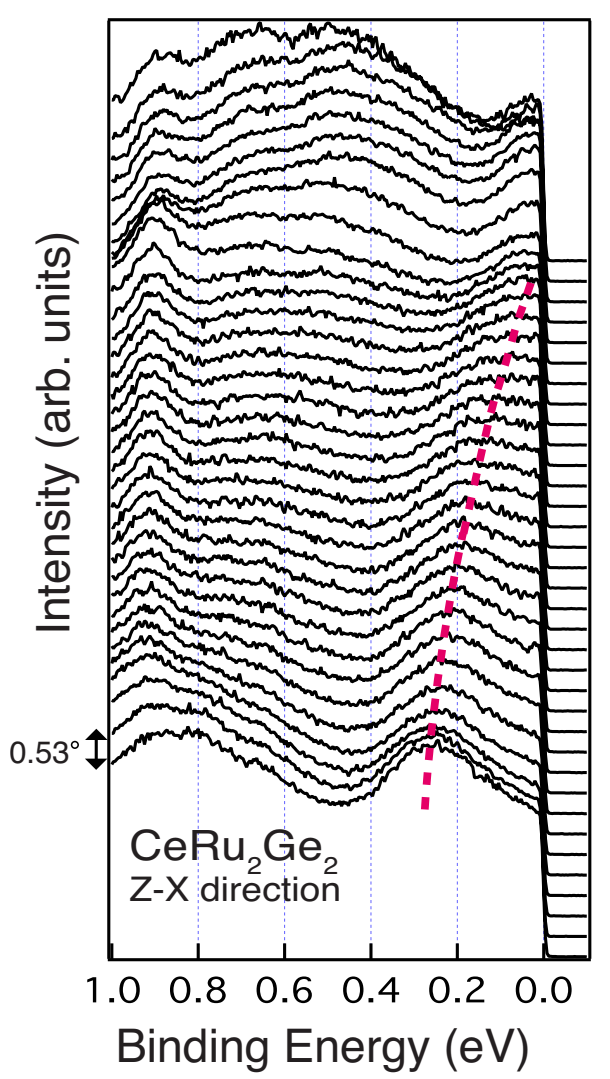

FIG. 11. (Color online) ELE-ARPES of an in situ cleaved $\mathrm{CeRu}_{2} \mathrm{Ge}_{2}$ at $3 \mathrm{~K}$ along the $\mathrm{z}$-x direction by using $\mathrm{Xe}+$ sapphire.

the spectral intensity at the $\mathrm{E}_{\mathrm{F}}$ is less than $1 / 2$ of the observed Fermi edge intensity. One further notices that with decreasing the temperature, the intensity at the $\mathrm{E}_{\mathrm{F}}$ decreases further. The DOS (not shown) obtained by dividing the ELEPES by FDDF clearly shows the opening of a gap with decreasing the temperature. Thus, this material is called a Kondo semiconductor. The DOS thus obtained have very similar shapes between 15 and $5 \mathrm{~K}$. In the case of $\mathrm{Yb}_{0.875} \mathrm{Lu}_{0.125} \mathrm{~B}_{12}$, the gap is shallower than $\mathrm{YbB}_{12}$ even at $5 \mathrm{~K}$. From the $\mathrm{Yb} 4 \mathrm{f}$ peak energy shifts observed by the bulk sensitive HAXPES (not shown), it is understood as the $\mathrm{Yb} 4 \mathrm{f}$ lattice coherence is suppressed by Lu doping by $12.5 \%$. Thus, the less prominent gap opening in $\mathrm{Yb}_{0.875} \mathrm{Lu}_{0.125} \mathrm{~B}_{12}$ is also understood as due to the collapse of the $4 \mathrm{f}$ lattice coherence and the ELEPES is understood to be again reflecting the bulk electronic structures in this case.

Now ELE-ARPES results obtained by the same system in Osaka University are shown for in situ cleaved $\mathrm{CeRu}_{2} \mathrm{Ge}_{2}$ at $3 \mathrm{~K}$ in the ferromagnetic phase. The results obtained by $\mathrm{Xe}$ lamp are shown in Fig. 11 for the $\mathrm{z}-\mathrm{x}$ direction with the angle step of $0.53^{\circ}$. A dispersing feature near the Fermi level is clearly traced.

Figure 12 summarizes the ELE-ARPES of a clean $\mathrm{Cu}(111)$ surface prepared by sputter-annealing technique. Measurements were performed at $90 \mathrm{~K}$ with the use of a pass voltage of $2 \mathrm{~V}$ of a SCIENTA SES200 analyzer in Jülich. The Xe spectrum took $\sim 5$ min with the use of a $0.2 \mathrm{~mm}$ slit, whereas the Ar spectrum took 35 min with the use of a $0.8 \mathrm{~mm}$ slit since the photon flux is much reduced by the $\mathrm{MgF}_{2}$ coating of the mirror. Meanwhile, the $\mathrm{Kr}$ spectrum carefully measured at 12 different sample rotation angles with the use of a $0.8 \mathrm{~mm}$ slit took $\sim 40 \mathrm{~min}$. A dispersing band approaching and crossing the $\mathrm{E}_{\mathrm{F}}$ is clearly seen for Xe, $\mathrm{Kr}$, and Ar excitations. It is known that the observed band originates from the surface state. ${ }^{1,18}$ In this configuration and energy region, there is no bulk state accessible by ELE-ARPES. The high counting rate means that ELE-ARPES is rather surface sensitive in this case. Since this technique is sometimes bulk sensitive and sometimes surface sensitive as demonstrated in the above examples, comparison with soft and hard $\mathrm{x}$-ray PES will be always helpful for understanding the real electronic structures.

\section{OUTLOOK}

It is demonstrated here that the ELEPES and ELEARPES by means of the microwave excited $\mathrm{Xe}, \mathrm{Kr}$, and $\mathrm{Ar}$ gases are as useful as those excited by the quasi-cw laser. The simple change of gas species without changing any essential part of the optical system is a great advantage of the use of such a light source for high accuracy spectroscopy. The availability of three different $\mathrm{h} \nu$ with resolution of better than $600 \mu \mathrm{eV}$ is superior to the laser system with only one strong $\mathrm{h} \nu$ in most cases. The matrix element effect and the bulk/surface sensitivity can be roughly studied by these three main lines. The lower price of the full lamp setup compared
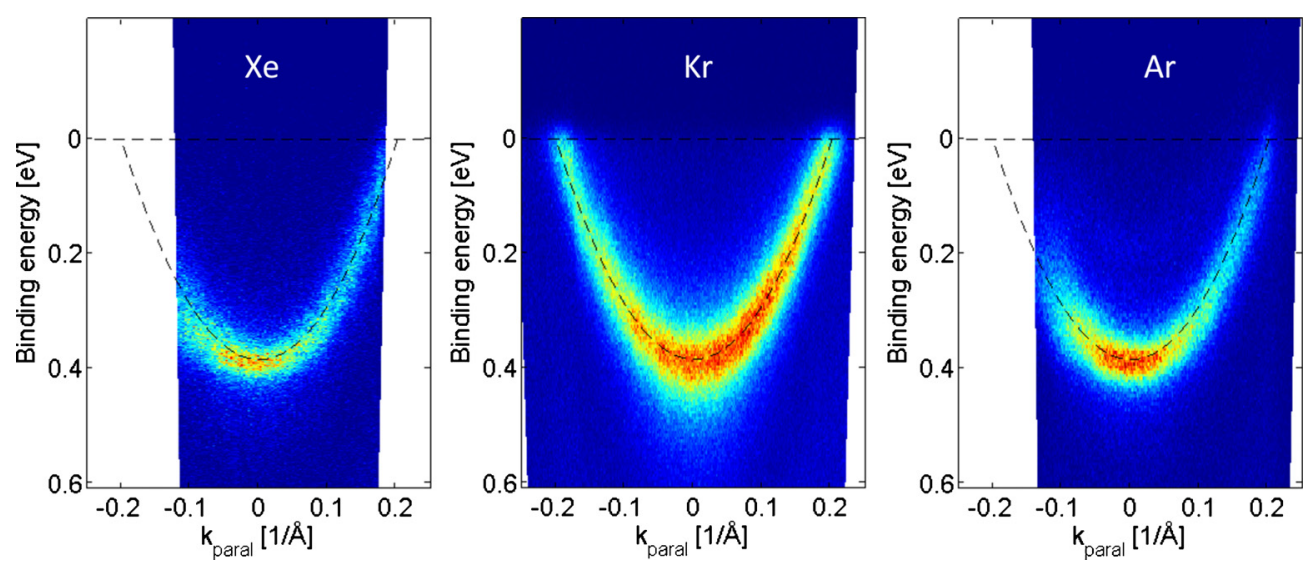

FIG. 12. (Color online) ELE-ARPES of a surface state measured on a sputter-annealed clean surface of $\mathrm{Cu}(111)$ at $90 \mathrm{~K}$. The pass voltage of $2 \mathrm{~V}$ is employed. 
with such a laser system is another advantage for many PES users. Polarization may be partly useful by setting the toroidal mirror at the Brewster angle, employing the different deviation angles, ${ }^{19}$ even though the polarization of the laser can be much higher than this method. Although the focusing down to $0.5 \mathrm{~mm}$ is of no practical difficulty, the focusing down to $0.1 \mathrm{~mm}$ is only possible at the sacrifice of the intensity. As shown here, the ELEPES and the ELE-ARPES can be bulk sensitive in some cases but are still surface sensitive in some other cases. In order to check its bulk sensitivity, it is always recommended to compare the results with those of SXPES, HAXPES, SX-ARPES, and HAX-ARPES. One typical style for a materials scientist may be to use the ELEPES and the ELE-ARPES in his/her own laboratory for the preliminary study. When one finds out some interesting materials and physics, further experiments will be performed with tunable synchrotron radiation not only in the comparable $\mathrm{h} \nu$ region but also in the slightly higher $\mathrm{h} \nu$ and $\mathrm{SX}$ regions to check the surface character. One may finally move to any high energy storage ring to perform HAXPES and HAX-ARPES. The combination of PES and ARPES in a wide energy range ${ }^{20}$ from a few $\mathrm{eV}$ up to $10000 \mathrm{eV}$ seems to be a very powerful approach to fully study the bulk and surface electronic structures of materials for fundamental sciences and/or for various applications.

Note added in proof. A qualitative photoemission work on a $\mathrm{LiF}$ filter applied to a conventional Ar light source was reported in Ref. 21.

\section{ACKNOWLEDGMENTS}

This work is supported by the Grand-in-Aid for Scientific Research from MEXT, Japan (Grant Nos. 21340101 and 21740229). S.S. is much obliged to the Alexander von Humboldt Foundation and Helmholtz Association for their support of his research stay in Germany.

\footnotetext{
${ }^{1}$ S. Hüfner, Photoelectron Spectroscopy, Principles and Applications (Springer, Berlin, 2003).

${ }^{2}$ A. Sekiyama, T. Iwasaki, K. Matsuda, Y. Saitoh, Y. Onuki, and S. Suga, Nature (London) 403, 396 (2000).
}

${ }^{3}$ S.-K. Mo, J. D. Denlinger, H.-D. Kim, H.-H. Park, J. W. Allen, A. Sekiyama, A. Yamasaki, K. Kadono, S. Suga, Y. Saitoh, T. Muro, P. Metcalf, G. Keller, K. Held, V. Eyert, V. I. Anisimov, and D. Vollhardt, Phys. Rev. Lett. 90, 186403 (2003).

${ }^{4}$ A. Sekiyama, H. Fujiwara, S. Imada, S. Suga, H. Eisaki, S. I. Uchida, K. Takegahara, H. Harima, Y. Saitoh, I. A. Nekrasov, G. Keller, D. E. Kondanov, A. V. Kozhevnikov, Th. Pruschke, K. Held, D. Vollhardt, and V. I. Anisimov, Phys. Rev. Lett. 93, 156402 (2004).

${ }^{5}$ S. Suga, A. Sekiyama, S. Imada, A. Shigemoto, A. Yamasaki, M. Tsunekawa, C. Dallera, L. Braicovich, T.-L. Lee, O. Sakai, T. Ebihara, and Y. Onuki, J. Phys. Soc. Jpn. 74, 2880 (2005).

${ }^{6}$ M. Taguchi, A. Chainani, K. Horiba, Y. Takata, M. Yabashi, K. Tamasaku, Y. Nishino, D. Miwa, T. Ishikawa, T. Takeuchi, K. Yamamoto, M. Matsunami, S. Shin, T. Yokoya, E. Ikenaga, K. Kobayashi, T. Mochiku, K. Hirata, J. Hori, K. Ishii, F. Nakamura, and T. Suzuki, Phys. Rev. Lett. 95, 177002 (2005)

${ }^{7}$ J. J. Yeh and I. Lindau, At. Data Nucl. Data Tables 32, 1 (1985).

${ }^{8}$ S. Suga, Appl. Phys. A: Mater. Sci. Process. 92, 479 (2008).

${ }^{9}$ Y. Takata, Y. Kayanuma, S. Oshima, S. Tanaka, M. Yabashi, K. Tamasaku, Y. Nishino, M. Matsunami, R. Eguchi, A. Chainani, M. Oura, T. Takeuchi, Y. Senba, H. Ohashi, S. Shin, and T. Ishikawa, Phys. Rev. Lett. 101, 137601 (2008).

${ }^{10}$ S. Suga, A. Sekiyama, H. Fujiwara, Y. Nakatsu, T. Miyamachi, S. Imada, P. Baltzer, S. Niitaka, H. Takagi, K. Yoshimura, M. Yabashi, K. Tamasaku, A. Higashiya, and T. Ishikawa, New J. Phys. 11, 073025 (2009).

${ }^{11}$ T. Kiss, F. Kanetaka, T. Yokota, T. Shimojima, K. Kanai, S. Shin, Y. Onuki, T. Togashi, C. Zhang, C. T. Chen, and S. Watanabe, Phys. Rev. Lett. 94, 057001 (2005).

${ }^{12}$ A. Shimoyamada, S. Tsuda, K. Ishizaka, T. Kiss, T. Shimojima, T. Togashi, S. Watanabe, C. Q. Zhang, C. T. Chen, Y. Matsushita, H. Ueda, Y. Ueda, and S. Shin, Phys. Rev. Lett. 96, 026403 (2006).

${ }^{13}$ T. Kiss, T. Shimojima, K. Ishizaka, A. Chainani, T. Togashi, T. Kanai, X.-Y. Wang, C.-T. Chen, S. Watanabe, and S. Shin, Rev. Sci. Instrum. 79, 023106 (2008).

${ }^{14}$ G. Funabashi, H. Fujiwara, A. Sekiyama, M. Hasumoto, T. Itoh, S. Kimura, P. Baltzer, and S. Suga, Jpn. J. Appl. Phys. 47, 2265 (2008).

${ }^{15}$ S. Souma, T. Sato, T. Takahashi, and P. Baltzer, Rev. Sci. Instrum. 78, 123104 (2007)

${ }^{16}$ H. Sumi and Y. Toyozawa, J. Phys. Soc. Jpn. 31, 342 (1971).

${ }^{17}$ S. Suga, A. Sekiyama, H. Fujiwara, Y. Nakatsu, J. Yamaguchi, M. Kimura, K. Murakami, S. Niitaka, H. Takagi, M. Yabashi, K. Tamasaku, A. Higashiya, T. Ishikawa, and I. Nekrasov, J. Phys. Soc. Jpn. 79, 044711 (2010).

${ }^{18}$ R. Paniago, R. Matzdorf, G. Meister, and A. Goldmann, Surf. Sci. 336, 113 (1995).

${ }^{19}$ J. A. Samson, Techniques of Vacuum Ultraviolet Spectroscopy (Wiley, New York, 1967).

${ }^{20}$ Very High Resolution Photoelectron Spectroscopy, Lecture Notes in Physics, edited by S. Hüfner (Springer, Berlin, 2007).

${ }^{21}$ M. Budke and M. Donath, Appl. Phys. Lett. 92, 231918 (2008). 